\title{
Whole-field measurement of three-dimensional stress by scattered-light photoelasticity with unpolarized light
}

\author{
Toshiki Kihara ${ }^{1, a}$ \\ ${ }^{1}$ School of Science and Engineering, Kinki University, Higashi-Osaka 577-8502, Japan
}

\begin{abstract}
In digital scattered-light photoelasticity with unpolarized light (DSLPUL), secondary principal stress direction $\psi_{\mathrm{j}}$ and total relative phase retardation $\rho_{\text {jtot }}$ in a three-dimensional stressed model with rotation of the principal stress axes are obtained by measuring Stokes parameters of scattered light from optical slices. The present paper describes intelligibly the principle of DSLPUL, and then demonstrates that the $\psi_{\mathrm{j}}$ and $\rho_{\text {jtot }}$ in a frozen stress sphere model are nondestructively measured over the entire field.
\end{abstract}

\section{Introduction}

Three-dimensional photoelasticity has encountered many difficulties for three-dimensional (3-D) stress analysis. This is mainly caused by the rotation of the principal stress directions along the light path. The optical phenomena occurring in a 3-D photoelastic model have been studied theoretically by many researchers [1-4], and it is well known that any 3-D photoelastic model can be reduced to an optically equivalent model consisting of a pure rotator and a linear retarder. These two sets of principal axes at the entrance and exit of the equivalent model are termed the primary and secondary characteristic directions, and the retardation is termed the characteristic phase retardation [2].

Three-dimensional Photoelasticity has been performed by the scattered-light method [4,7-22], the speckle technique method [23,24], integrated photoelasticity [4-6], and the frozen stress method [25], which is a destructive method. Up to the present time it is difficult to measure nondestructively the stress state in a 3-D photoelasticity with general rotation of the principal stress axes except for the scattered-light photoelasticity. In the scattered-light photoelasticity, there are two scattered-light methods. One [4,7-11] is the conventional scattered-light method with scattered light used as an analyzer that polarized light is used as the incident light. The scattered light is directly observed from a direction perpendicular to the incident light beam, and stress data is obtained along the incident light path. The other [4,12-22] is the scattered-light photoelasticity with scattered light used as a polarizer, unpolarized light is usually used as the incident light. It is called scattered-light photoelasticity using unpolarized light (SLPUL), and the photoelastic effect of the incident path is eliminated, while the photoelastic effect of the light path in the direction of observation is examined through an analysis system.

I have derived a SLPUL method for measuring $\rho_{\mathrm{j}}$ and $\psi_{\mathrm{j}}$ in a 3-D photoelastic model that has undergone principal stress rotation [17-19]. Then a technique for automatic stress analysis of the method, digital scattered-light photoelasticity with unpolarized light (DSLPUL), is proposed and

a e-mail : kihara@mech.kindai.ac.jp

This is an Open Access article distributed under the terms of the Creative Commons Attribution-Noncommercial License 3.0, which permits unrestricted use, distribution, and reproduction in any noncommercial medium, provided the original work is properly cited. 
developed, in which values of $\psi_{\mathrm{j}}$ and the total relative phase retardation $\rho_{\mathrm{jtot}}$ in a 3-D photoelastic model can be obtained over the entire field from the arctangent function, and the technique was validated through numerical simulations and experiments [21,22].

The present paper shows that the principle of DSLPUL is intelligibly described and the usefulness of the method is shown with nondestructive experiment results of the $\rho_{\text {jtot }}$ and $\psi_{\mathrm{j}}$ in a frozen stress sphere model.

\section{Relations between photoelastic parameters and Stokes parameters in three-dimensional photoelasticity}

A schematic diagram of DSLPUL [17,20] is shown in Figure 1. In the figure, an unpolarized light impinges at a $\theta$-deg angle to the $\mathrm{x}$ axis in the $x-z$ plane at the point $y_{\mathrm{j}}$ in the model. The resultant scattered light, $\boldsymbol{S}\left(y_{\mathrm{j}} ; \theta\right)$, at the point $y_{\mathrm{j}}$ is observed by an analysis system, which consists of a quarter-wave plate $Q_{2}$, and a linear polarizer $P_{2}$. The quantity $\boldsymbol{S}\left(y_{\mathrm{j}} ; \theta\right)$ is the Stokes vector of the scattered light, and is the linearly polarized light of the azimuth (the $\theta$-deg axis) perpendicular to both the incident light direction and the observation direction [15]. The state of polarization of the scattered light is then changed by the photoelastic model $R\left(2 \omega_{\mathrm{j}-1,0}\right) M_{\mathrm{j}-1,0} M_{\mathrm{j}}$ between points $y_{\mathrm{j}}$ and $y_{0}$. Here, $M_{\mathrm{j}}$ is the Mueller matrix of the linear retarder with relative phase retardation $\rho_{\mathrm{j}}$ and secondary principal stress directions $\left(\psi_{\mathrm{j}}\right.$, $\left.\psi_{\mathrm{j}}+\pi / 2\right)$ between the points $y_{\mathrm{j}}$ and $y_{\mathrm{j}-1}$. The matrices $R\left(2 \omega_{\mathrm{j}-1,0}\right)$ and $M_{\mathrm{j}-1,0}$ are the Mueller matrices of a pure rotator with rotatory power $\omega_{\mathrm{j}-}$

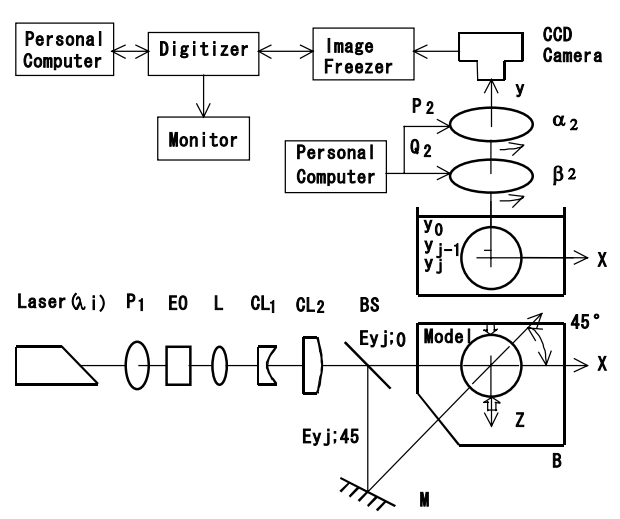

Fig.1. Schematic diagram showing optical arrangement and image processing system used in experiments. EO: electro-optical modulation device, $C L_{1}$ and $C L_{2}$ : cylindrical lenses, $B$ : immersion cell, $Q_{2}$ : quarter-wave plate, $P_{1}$ and $P_{2}$ : linear polarizers, BS: beam splitter.

${ }_{1,0}$, and of a linear retarder with retardation $\rho_{\mathrm{j}-1,0}$ and principal axes of azimuths $\left(\psi_{\mathrm{j}-1,0}, \psi_{\mathrm{j}-1,0}+\pi / 2\right)$, respectively, for the optically equivalent model between point $y_{\mathrm{j}-1}$ and $y_{0}$. The Stokes vector $\boldsymbol{S}\left(y_{\mathrm{j}}, y_{0} ; \theta\right)$ of the observed light can therefore be expressed by the following matrix equation $[4,21]$.

$\boldsymbol{S}\left(y_{\mathrm{j}}, y_{0} ; \theta\right)=U\left(y_{\mathrm{j}-1}, y_{0}\right) M_{\mathrm{j}} \boldsymbol{S}\left(y_{\mathrm{j}} ; \theta\right)$

where

$$
\begin{aligned}
& U\left(y_{\mathrm{j}-1}, y_{0}\right)=R\left(2 \omega_{\mathrm{j}-1,0}\right) M_{\mathrm{j}-1,0}, \\
& R\left(2 \omega_{\mathrm{j}-1,0}\right)=\left[\begin{array}{cccc}
1 & 0 & 0 & 0 \\
0 & \cos 2 \omega_{\mathrm{j}-1,0} & -\sin 2 \omega_{\mathrm{j}-1,0} & 0 \\
0 & \sin 2 \omega_{\mathrm{j}-1,0} & \cos 2 \omega_{\mathrm{j}-1,0} & 0 \\
0 & 0 & 0 & 1
\end{array}\right] \text {, } \\
& M_{\mathrm{j}-1,0}=\left[\begin{array}{cccc}
1 & 0 & 0 & 0 \\
1 & -\left(1-\cos \rho_{\mathrm{j}-1,0}\right) \sin ^{2} 2 \psi_{\mathrm{j}-1,0} & \left(1-\cos \rho_{\mathrm{j}-1,0}\right) \sin 2 \psi_{\mathrm{j}-1,0} \cos 2 \psi_{\mathrm{j}-1,0} & -\sin \rho_{\mathrm{j}-1,0} \sin 2 \psi_{\mathrm{j}-1,0} \\
0 & \left(1-\cos \rho_{\mathrm{j}-1,0}\right) \sin 2 \psi_{\mathrm{j}-1,0} \cos 2 \psi_{\mathrm{j}-1,0} & 1-\left(1-\cos \rho_{\mathrm{j}-1,0}\right) \cos ^{2} 2 \psi_{\mathrm{j}-1,0} & \sin \rho_{\mathrm{j}-1,0} \cos 2 \psi_{\mathrm{j}-1,0} \\
0 & \sin \rho_{\mathrm{j}-1,0} \sin 2 \psi_{\mathrm{j}-1,0} & -\sin \rho_{\mathrm{j}-1,0} \cos 2 \psi_{\mathrm{j}-1,0} & \cos \rho_{\mathrm{j}-1,0}
\end{array}\right] \\
& M_{\mathrm{j}}=\left[\begin{array}{cccc}
1 & 0 & 0 & 0 \\
0 & 1-\left(1-\cos \rho_{\mathrm{j}}\right) \sin ^{2} 2 \psi_{\mathrm{j}} & \left(1-\cos \rho_{\mathrm{j}}\right) \sin 2 \psi_{\mathrm{j}} \cos 2 \psi_{\mathrm{j}} & -\sin \rho_{\mathrm{j}} \sin 2 \psi_{\mathrm{j}} \\
0 & \left(1-\cos \rho_{\mathrm{j}}\right) \sin 2 \psi_{\mathrm{j}} \cos 2 \psi_{\mathrm{j}} & 1-\left(1-\cos \rho_{\mathrm{j}}\right) \cos ^{2} 2 \psi_{\mathrm{j}} & \sin \rho_{\mathrm{j}} \cos 2 \psi_{\mathrm{j}} \\
0 & \sin \rho_{\mathrm{j}} \sin 2 \psi_{\mathrm{j}} & -\sin \rho_{\mathrm{j}} \cos 2 \psi_{\mathrm{j}} & \cos \rho_{\mathrm{j}}
\end{array}\right],
\end{aligned}
$$


14th International Conference on Experimental Mechanics

$\boldsymbol{S}\left(y_{\mathrm{j}} ; \theta\right)=\boldsymbol{S}_{\mathbf{0}}\left(y_{\mathrm{j}} ; \theta\right)\left[\begin{array}{c}1 \\ \cos 2 \theta \\ \sin 2 \theta \\ 0\end{array}\right]$

Similarly, if unpolarized light impinges at the point $y_{\mathrm{j}-1}$, the scattered light $\boldsymbol{S}\left(y_{\mathrm{j}-1} ; \theta\right)$ is also the linearly polarized light of the azimuth $\theta$. The observed light vector can be expressed as

$\boldsymbol{S}\left(y_{\mathrm{j}-1}, y_{0} ; \theta\right)=U\left(y_{\mathrm{j}-1}, y_{0}\right) \boldsymbol{S}\left(y_{\mathrm{j}-1} ; \theta\right)$

By multiplying both members of Eq. (1) by the inverse matrix $U\left(y_{\mathrm{j}-1}, y_{0}\right)^{-1}=M_{\mathrm{j}-1,0}{ }^{-1} R\left(2 \omega_{\mathrm{j}-1,0}\right)^{-1}$, the following equations are obtained by representation for the normalized Stokes vectors as

$U\left(y_{\mathrm{j}-1}, y_{0}\right)^{-1} \boldsymbol{S}\left(y_{\mathrm{j}}, y_{0} ; \theta\right) / S_{0}\left(y_{\mathrm{j}}, y_{0} ; \theta\right)=M_{\mathrm{j}} \boldsymbol{S}\left(y_{\mathrm{j}} ; \theta\right) / S_{0}\left(y_{\mathrm{j}} ; \theta\right)$.

Where

$$
U\left(y_{\mathrm{j}-1}, y_{0}\right)^{-1}=\left[\begin{array}{cc}
1 & 0 \\
0 & \cos 2 \psi_{\mathrm{j}-1,0} \cos \left(2 \psi_{\mathrm{j}-1,0}+2 \omega_{\mathrm{j}-1,0}\right)+\cos \rho_{\mathrm{j}-1,0} \sin 2 \psi_{\mathrm{j}-1,0} \sin \left(2 \psi_{\mathrm{j}-1,0}+2 \omega_{\mathrm{j}-1,0}\right) \\
0 & \sin 2 \psi_{\mathrm{j}-1,0} \cos \left(2 \psi_{\mathrm{j}-1,0}+2 \omega_{\mathrm{j}-1,0}\right)-\cos \rho_{\mathrm{j}-1,0} \cos 2 \psi_{\mathrm{j}-1,0} \sin \left(2 \psi_{\mathrm{j}-1,0}+2 \omega_{\mathrm{j}-1,0}\right) \\
0 & -\sin \rho_{\mathrm{j}-1,0} \sin \left(2 \psi_{\mathrm{j}-1,0}+2 \omega_{\mathrm{j}-1,0}\right)
\end{array}\right.
$$

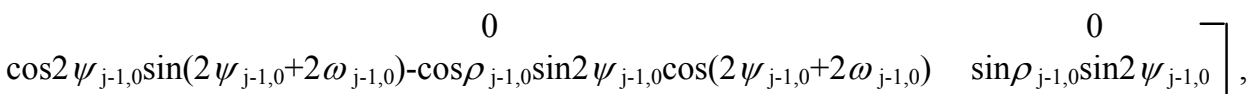
$\sin 2 \psi_{\mathrm{j}-1,0} \sin \left(2 \psi_{\mathrm{j}-1,0}+2 \omega_{\mathrm{j}-1,0}\right)+\cos \rho_{\mathrm{j}-1,0} \cos 2 \psi_{\mathrm{j}-1,0} \cos \left(2 \psi_{\mathrm{j}-1,0}+2 \omega_{\mathrm{j}-1,0}\right)-\sin \rho_{\mathrm{j}-1,0} \cos 2 \psi_{\mathrm{j}-1,0}$ $\sin \rho_{\mathrm{j}-1,0} \cos \left(2 \psi_{\mathrm{j}-1,0}+2 \omega_{\mathrm{j}-1,0}\right)$

$\cos \rho_{\mathrm{j}-1,0}$

$$
=\left[\begin{array}{cccc}
1 & 0 & 0 & 0 \\
0 & s_{1}\left(y_{\mathrm{j}-1}, y_{0} ; 0\right) & s_{2}\left(y_{\mathrm{j}-1}, y_{0} ; 0\right) & s_{3}\left(y_{\mathrm{j}-1}, y_{0} ; 0\right) \\
0 & s_{1}\left(y_{\mathrm{j}-1}, y_{0} ; 45\right) & s_{2}\left(y_{\mathrm{j}-1}, y_{0} ; 45\right) & s_{3}\left(y_{\mathrm{j}-1}, y_{0} ; 45\right) \\
0 & U\left(y_{\mathrm{j}-1}, y_{0}\right)^{-1} 42 & U\left(y_{\mathrm{j}-1}, y_{0}\right)^{-1} 43 & U\left(y_{\mathrm{j}-1}, y_{0}\right)^{-1} 44
\end{array}\right] \text {. }
$$

Here, $s\left(y_{\mathrm{j}-1}, y_{0} ; \theta\right)$ is the normalized polarization vector $\left\{S_{1}\left(y_{\mathrm{j}-1}, y_{0} ; \theta\right) / S_{0}\left(y_{\mathrm{j}-1}, y_{0} ; \theta\right), S_{2}\left(y_{\mathrm{j}-1}, y_{0} ; \theta\right) / S_{0}\left(y_{\mathrm{j}-}\right.\right.$ $\left.\left.{ }_{1}, y_{0} ; \theta\right), S_{3}\left(y_{\mathrm{j}-1}, y_{0} ; \theta\right) / S_{0}\left(y_{\mathrm{j}-1}, y_{0} ; \theta\right)\right\}$ [26]. On the other hand, the quantity $U\left(y_{\mathrm{j}-1}, y_{0}\right)^{-1}{ }_{\mathrm{kl}}$ is the matrix element of $U\left(y_{\mathrm{j}-1}, y_{0}\right)^{-1}$, where the subscripts $k$ and $l$ take the values of $k=1,2,3,4$ and $l=1,2,3,4$.

The inverse matrix elements $U\left(y_{\mathrm{j}-1}, y_{0}\right)^{-1}{ }_{42}, U\left(y_{\mathrm{j}-1}, y_{0}\right)^{-1}{ }_{43}$, and $U\left(y_{\mathrm{j}-1}, y_{0}\right)^{-1}{ }_{44}$ are given from Eq. (9).

$$
\begin{aligned}
U\left(y_{\mathrm{j}-1}, y_{0}\right)^{-1}{ }_{42} & =-\sin \rho_{\mathrm{j}-1,0} \sin \left(2 \psi_{\mathrm{j}-1,0}+2 \omega_{\mathrm{j}-1,0}\right) \\
& =-s_{3}\left(y_{\mathrm{j}-1}, y_{0} ; 0\right) \cos 2 \omega_{\mathrm{j}-1,0}+s_{3}\left(y_{\mathrm{j}-1}, y_{0} ; 45\right) \sin 2 \omega_{\mathrm{j}-1,0}, \\
U\left(y_{\mathrm{j}-1}, y_{0}\right)^{-1}{ }_{43} & =\cos \left(2 \psi_{\mathrm{j}-1,0}+2 \omega_{\mathrm{j}-1,0}\right) \\
& =-s_{3}\left(y_{\mathrm{j}-1}, y_{0} ; 45\right) \cos 2 \omega_{\mathrm{j}-1,0}-s_{3}\left(y_{\mathrm{j}-1}, y_{0} ; 0\right) \sin 2 \omega_{\mathrm{j}-1,0}, \\
U\left(y_{\mathrm{j}-1}, y_{0}\right)^{-1}{ }_{44} & =\cos \rho_{\mathrm{j}-1,0} \\
& =\left[s_{1}\left(y_{\mathrm{j}-1}, y_{0} ; 0\right)+s_{2}\left(y_{\mathrm{j}-1}, y_{0} ; 45\right)\right] \cos 2 \omega_{\mathrm{j}-1,0}+\left[s_{2}\left(y_{\mathrm{j}-1}, y_{0} ; 0\right)-s_{1}\left(y_{\mathrm{j}-1}, y_{0} ; 45\right)\right] \sin 2 \omega_{\mathrm{j}-1,0}-1 .
\end{aligned}
$$

The terms $\sin 2 \omega_{\mathrm{j}-1,0}$ and $\cos 2 \omega_{\mathrm{j}-1,0}$ are also given as followings [21]:

$$
\begin{aligned}
\sin 2 \omega_{\mathrm{j}-1,0} & =\left\{\left[s_{2}\left(y_{\mathrm{j}-1}, y_{0} ; 0\right)-s_{1}\left(y_{\mathrm{j}-1}, y_{0} ; 45\right)\right]\right\} /\left\{\left[s_{2}\left(y_{\mathrm{j}-1}, y_{0} ; 0\right)-s_{1}\left(y_{\mathrm{j}-1}, y_{0} ; 45\right)\right]^{2}+\left[s_{1}\left(y_{\mathrm{j}-1}, y_{0} ; 0\right)+s_{2}\left(y_{\mathrm{j}-1}, y_{0} ; 45\right)\right]^{2}\right\}^{0.5} \\
& =\left[2 \sin 2 \omega_{\mathrm{j}-1,0} \cos ^{2}\left(\rho_{\mathrm{j}-1,0} / 2\right)\right] /\left[2 \cos ^{2}\left(\rho_{\mathrm{j}-1,0} / 2\right)\right] . \\
\cos 2 \omega_{\mathrm{j}-1,0} & =\left\{\left[s_{1}\left(y_{\mathrm{j}-1}, y_{0} ; 0\right)+s_{2}\left(y_{\mathrm{j}-1}, y_{0} ; 45\right)\right]\right\} /\left\{\left[s_{2}\left(y_{\mathrm{j}-1}, y_{0} ; 0\right)-s_{1}\left(y_{\mathrm{j}-1}, y_{0} ; 45\right)\right]^{2}+\left[s_{1}\left(y_{\mathrm{j}-1}, y_{0} ; 0\right)+s_{2}\left(y_{\mathrm{j}-1}, y_{0} ; 45\right)\right]^{2}\right\}^{0.5} \\
& =\left[2 \cos 2 \omega_{\mathrm{j}-1,0} \cos ^{2}\left(\rho_{\mathrm{j}-1,0} / 2\right)\right] /\left[2 \cos ^{2}\left(\rho_{\mathrm{j}-1,0} / 2\right)\right] .
\end{aligned}
$$

Then, by separately impinging two unpolarized lights oriented at $0^{\circ}$ and $45^{\circ}$, respectively, the left side of Eq. (8) can be measured and we may express them, respectively, as

$$
\begin{aligned}
U\left(y_{\mathrm{j}-1}, y_{0}\right)^{-1} \boldsymbol{s}\left(y_{\mathrm{j}}, y_{0} ; 0\right) & =\left[\begin{array}{c}
1 \\
\boldsymbol{s}\left(y_{\mathrm{j}-1}, y_{0} ; 0\right) \boldsymbol{s}\left(y_{\mathrm{j}}, y_{0} ; 0\right) \\
\boldsymbol{s}\left(y_{\mathrm{j}-1}, y_{0} ; 45\right) \boldsymbol{s}\left(y_{\mathrm{j}}, y_{0} ; 0\right)
\end{array}\right. \\
& =\boldsymbol{s}\left(y_{\mathrm{j}-1}, y_{\mathrm{j}} ; 0\right),
\end{aligned}
$$




$$
\begin{aligned}
U\left(y_{\mathrm{j}-1}, y_{0}\right)^{-1} \boldsymbol{s}\left(y_{\mathrm{j}}, y_{0} ; 45\right) & =\left[\begin{array}{c}
1 \\
\boldsymbol{s}\left(y_{\mathrm{j}-1}, y_{0} ; 0\right) \boldsymbol{s}\left(y_{\mathrm{j}}, y_{0} ; 45\right) \\
\boldsymbol{s}\left(y_{\mathrm{j}-1}, y_{0} ; 45\right) \boldsymbol{s}\left(y_{\mathrm{j}}, y_{0} ; 45\right) \\
\left(U\left(y_{\mathrm{j}-1}, y_{0}\right)^{-1}{ }_{42}, U\left(y_{\mathrm{j}-1}, y_{0}\right)^{-1}{ }_{43}, U\left(y_{\mathrm{j}-1}, y_{0}\right)^{-1}{ }_{44}\right) \boldsymbol{s}\left(y_{\mathrm{j}}, y_{0} ; 45\right)
\end{array}\right] \\
& =\boldsymbol{s}\left(y_{\mathrm{j}-1}, y_{\mathrm{j}} ; 45\right) .
\end{aligned}
$$

where, $\boldsymbol{s}\left(y_{\mathrm{j}-1}, y_{0} ; 0\right) \boldsymbol{s}\left(y_{j}, y_{0} ; 0\right)$ is the inner product of the normalized polarization vectors. Since the right side of Eq. (8) is associated with $\psi_{\mathrm{j}}$ and $\rho_{\mathrm{j}}$, Eq.(8) is expressed, respectively, as

$$
\begin{aligned}
& \boldsymbol{s}\left(y_{\mathrm{j}-1}, y_{\mathrm{j}} ; 0\right)=\left[\begin{array}{c}
1 \\
1-\left(1-\cos \rho_{\mathrm{j}}\right) \sin ^{2} 2 \psi_{\mathrm{j}} \\
\left(1-\cos \rho_{\mathrm{j}}\right) \sin 2 \psi_{\mathrm{j}} \cos 2 \psi_{\mathrm{j}} \\
\sin \rho_{\mathrm{j}} \sin 2 \psi_{\mathrm{j}}
\end{array}\right], \\
& \boldsymbol{s}\left(y_{\mathrm{j}-1}, y_{\mathrm{j}} ; 45\right)=\left[\begin{array}{c}
1 \\
\left(1-\cos \rho_{\mathrm{j}}\right) \sin 2 \psi_{\mathrm{j}} \cos 2 \psi_{\mathrm{j}} \\
1-\left(1-\cos \rho_{\mathrm{j}}\right) \cos ^{2} 2 \psi_{\mathrm{j}} \\
-\sin \rho_{\mathrm{j}} \cos 2 \psi_{\mathrm{j}}
\end{array}\right] .
\end{aligned}
$$

These Eqs. (17) and (18) are the same expresions as those of two-dimensional photoelasticity's analysis [28].

The values of $\psi_{\mathrm{j}}$ and $\rho_{\mathrm{j}}$ in the optical slice between the points $y_{\mathrm{j}}$ and $y_{\mathrm{j}-1}$ are derived from Eqs. (9)-(12) [21]:

$$
\begin{aligned}
\psi_{\mathrm{j}} & =0.25 \tan ^{-1}\left\{\left[\mathrm{~s}_{2}\left(y_{\mathrm{j}-1}, y_{\mathrm{j}} ; 0\right)+\mathrm{s}_{1}\left(y_{\mathrm{j}-1}, y_{\mathrm{j}} ; 45\right)\right] /\left[\mathrm{s}_{1}\left(y_{\mathrm{j}-1}, y_{\mathrm{j}} ; 0\right)-\mathrm{s}_{2}\left(y_{\mathrm{j}-1}, y_{\mathrm{j}} ; 45\right)\right]\right\} \\
& =0.25 \tan ^{-1}\left\{\left[2 \sin ^{2}\left(\rho_{\mathrm{j}} / 2\right) \sin 4 \psi_{\mathrm{j}}\right] /\left[2 \sin ^{2}\left(\rho_{\mathrm{j}} / 2\right) \cos 4 \psi_{\mathrm{j}}\right]\right\} \\
\rho_{\mathrm{j}} & =\tan ^{-1}\left\{\left[\mathrm{~s}_{3}\left(y_{\mathrm{j}-1}, y_{\mathrm{j}} ; 0\right) \sin 2 \psi_{\mathrm{j}}-\mathrm{s}_{3}\left(y_{\mathrm{j}-1}, y_{\mathrm{j}} ; 45\right) \cos 2 \psi_{\mathrm{j}}\right] /\left[\mathrm{s}_{1}\left(y_{\mathrm{j}-1}, y_{\mathrm{j}} ; 0\right)+\mathrm{s}_{2}\left(y_{\mathrm{j}-1}, y_{\mathrm{j}} ; 45\right)-1\right]\right\} \\
& =\tan ^{-1}\left[\sin \rho_{\mathrm{j}}\left(\sin ^{2} 2 \psi_{\mathrm{j}}+\cos ^{2} 2 \psi_{\mathrm{j}}\right) / \cos \rho_{\mathrm{j}}\right] .
\end{aligned}
$$

The measurement range of the angle $\psi_{\mathrm{j}}$ is $(-\pi / 8, \pi / 8)$; however, $\psi_{\mathrm{j}}$ is undefined when $\rho_{\mathrm{jtot}}=2 \pi N$, where $N$ is an integer. The measurement range of the angle $\rho_{\mathrm{j}}$ is $(-\pi / 2, \pi / 2)$.

\section{Determination of $\psi_{\mathrm{j}}$ and $\rho_{\mathrm{j} t \mathrm{tot} \mathrm{i}}$ by use of Three Wavelengths}

\subsection{Determination of $\psi_{\mathrm{j}}$}

From Eq. (19), the value of $\psi_{\mathrm{j}}$ can be measured more accurately when the term $\sin ^{2}\left(\rho_{\mathrm{j}} / 2\right)$ is larger. The value of $\psi_{j}$ can be determined from Eq. (21) using images measured at three different wavelengths $\left(\lambda_{1}, \lambda_{2}, \lambda_{3}\right)$ selected appropriately as follows [28].

$$
\begin{gathered}
\psi_{\mathrm{j}}=0.25 \tan ^{-1}\left\{\left[\left(\mathrm{~s}_{2}\left(y_{\mathrm{j}-1}, y_{\mathrm{j}} ; 0\right)+\mathrm{s}_{1}\left(y_{\mathrm{j}-1}, y_{\mathrm{j}} ; 45\right)\right)_{\lambda 1}+\left(\mathrm{s}_{2}\left(y_{\mathrm{j}-1}, y_{\mathrm{j}} ; 0\right)+\mathrm{s}_{1}\left(y_{\mathrm{j}-1}, y_{\mathrm{j}} ; 45\right)\right)_{\lambda 2}+\left(\mathrm{s}_{2}\left(y_{\mathrm{j}-1}, y_{\mathrm{j}} ; 0\right)+\mathrm{s}_{1}\left(y_{\mathrm{j}-1}, y_{\mathrm{j}} ; 45\right)\right)_{\lambda 3}\right]\right. \\
\left.\left.\left.\quad /\left[\left(\mathrm{s}_{1}\left(y_{\mathrm{j}-1}, y_{\mathrm{j}} ; 0\right)-\mathrm{s}_{2}\left(y_{\mathrm{j}-1}, y_{\mathrm{j}} ; 45\right)\right)\right)_{\lambda 1}+\left(\mathrm{s}_{1}\left(y_{\mathrm{j}-1}, y_{\mathrm{j}} ; 0\right)-\mathrm{s}_{2}\left(y_{\mathrm{j}-1}, y_{\mathrm{j}} ; 45\right)\right)\right)_{\lambda 2}-\left(\mathrm{s}_{1}\left(y_{\mathrm{j}-1}, y_{\mathrm{j}} ; 0\right)+\mathrm{s}_{2}\left(y_{\mathrm{j}-1}, y_{\mathrm{j}} ; 45\right)\right)_{\lambda 3}\right]\right\} \\
=0.25 \tan ^{-1}\left\{\left[\left(\sin ^{2}\left(\rho_{\mathrm{j} \lambda 1} / 2\right)+\sin ^{2}\left(\rho_{\mathrm{j} \lambda 2} / 2\right)+\sin ^{2}\left(\rho_{\mathrm{j} \lambda} / 2\right)\right) 2 \sin 4 \psi_{\mathrm{j}}\right]\right. \\
\left./\left[\left(\sin ^{2}\left(\rho_{\mathrm{j} \lambda 1} / 2\right)+\sin ^{2}\left(\rho_{\mathrm{j} \lambda 2} / 2\right)+\sin ^{2}\left(\rho_{\mathrm{j} \lambda} / 2\right)\right) 2 \cos 4 \psi_{\mathrm{j}}\right]\right\}
\end{gathered}
$$

Here, $\left(\mathrm{s}_{2}\left(y_{\mathrm{j}-1}, y_{\mathrm{j}} ; 0\right)+\mathrm{s}_{1}\left(y_{\mathrm{j}-1}, y_{\mathrm{j}} ; 45\right)\right)_{\lambda \mathrm{i}}$ denotes $\left(\mathrm{s}_{2}\left(y_{\mathrm{j}-1}, y_{\mathrm{j}} ; 0\right)+\mathrm{s}_{1}\left(y_{\mathrm{j}-1}, y_{\mathrm{j}} ; 45\right)\right)$ at $\lambda_{\mathrm{i}}$, and $\rho_{\mathrm{j}-1,0 \lambda \mathrm{i}}$ denotes $\rho_{\mathrm{j}-1,0}$ at $\lambda_{\mathrm{i}}$. The value of $\psi_{\mathrm{j}}$ given by Eq. (21) can compensates for influence of $\rho_{\mathrm{jtot} \lambda \mathrm{i}}=2 \pi N_{\lambda_{\mathrm{i}}}$ by using three wavelengths where $N_{\lambda_{\mathrm{i}}}$ is $N$ at $\lambda_{\mathrm{i}}$. The expansion of the measurement range of $\psi_{\mathrm{j}}$ to $(-\pi / 2, \pi / 2)$ is referred to as the phase unwrapping of $\psi_{\mathrm{j}}$, and is performed by eliminating the phase jumps at $\pi / 4$ or $-\pi / 4$ for $\psi_{j}$.

\subsection{Determination of $\rho_{\mathrm{jtot} \lambda \mathrm{i}}$}


The value of $\rho_{\mathrm{j} \lambda \mathrm{i}}$ is calculated from Eq. (20), in which $\rho_{\mathrm{j} \lambda \mathrm{i}} \operatorname{contains} \sin 2 \omega_{\mathrm{j}-1,0}$ and $\cos 2 \omega_{\mathrm{j}-1,0}$. These $\sin 2 \omega_{\mathrm{j}-1,0}$ and $\cos 2 \omega_{\mathrm{j}-1,0}$ are determined from Eqs. (22) and (23) using the three wavelengths $\left(\lambda_{1}, \lambda_{2}, \lambda_{3}\right)$ as follows.

$$
\begin{aligned}
& \sin 2 \omega_{\mathrm{j}-1,0}=\left\{\left[s_{2}\left(y_{\mathrm{j}-1}, y_{0} ; 0\right)-s_{1}\left(y_{\mathrm{j}-1}, y_{0} ; 45\right)\right] \lambda_{\lambda 1}+\left[s_{2}\left(y_{\mathrm{j}-1}, y_{0} ; 0\right)-s_{1}\left(y_{\mathrm{j}-1}, y_{0} ; 45\right)\right]_{\lambda 2}+\left[s_{2}\left(y_{\mathrm{j}-1}, y_{0} ; 0\right)-s_{1}\left(y_{\mathrm{j}-1}, y_{0} ; 45\right)\right]_{\lambda 3}\right\} \\
& /\left\{\left[\left(s_{2}\left(y_{\mathrm{j}-1}, y_{0} ; 0\right)-s_{1}\left(y_{\mathrm{j}-1}, y_{0} ; 45\right)\right)^{2}+\left(s_{1}\left(y_{\mathrm{j}-1}, y_{0} ; 0\right)+s_{2}\left(y_{\mathrm{j}-1}, y_{0} ; 45\right)\right)^{2}\right]^{0.5} \lambda_{1}+[]_{\lambda 2}+[]_{\lambda 3}\right\} \\
&=\left\{2 \sin 2 \omega_{\mathrm{j}-1,0}\left[\cos ^{2}\left(\rho_{\mathrm{j}-1,0 \lambda 1} / 2\right)+\cos ^{2}\left(\rho_{\mathrm{j}-1,0 \lambda 2} / 2\right)+\cos ^{2}\left(\rho_{\mathrm{j}-1,0 \lambda 3} / 2\right)\right]\right\} \\
& /\left\{2\left[\cos ^{2}\left(\rho_{\mathrm{j}-1,0 \lambda 1} / 2\right)+\cos ^{2}\left(\rho_{\mathrm{j}-1,0 \lambda 2} / 2\right)+\cos ^{2}\left(\rho_{\mathrm{j}-1,0 \lambda 3} / 2\right)\right]\right\}, \\
& \cos 2 \omega_{\mathrm{j}-1,0}=\left\{\left[s_{1}\left(y_{\mathrm{j}-1}, y_{0} ; 0\right)+s_{2}\left(y_{\mathrm{j}-1}, y_{0} ; 45\right)\right]_{\lambda 1}+\left[s_{1}\left(y_{\mathrm{j}-1}, y_{0} ; 0\right)+s_{2}\left(y_{\mathrm{j}-1}, y_{0} ; 45\right)\right]_{\lambda 2}+\left[s_{1}\left(y_{\mathrm{j}-1}, y_{0} ; 0\right)+s_{2}\left(y_{\mathrm{j}-1}, y_{0} ; 45\right)\right]_{\lambda 3}\right\} \\
& /\left\{\left[\left(s_{2}\left(y_{\mathrm{j}-1}, y_{0} ; 0\right)-s_{1}\left(y_{\mathrm{j}-1}, y_{0} ; 45\right)\right)^{2}+\left(s_{1}\left(y_{\mathrm{j}-1}, y_{0} ; 0\right)+s_{2}\left(y_{\mathrm{j}-1}, y_{0} ; 45\right)\right)^{2}\right]^{0.5}{ }_{\lambda 1}+[]_{\lambda 2}+[]_{\lambda 3}\right\} \\
&=\left\{2 \cos 2 \omega_{\mathrm{j}-1,0}\left[\cos ^{2}\left(\rho_{\mathrm{j}-1,0 \lambda 1} / 2\right)+\cos ^{2}\left(\rho_{\mathrm{j}-1,0 \lambda 2} / 2\right)+\cos ^{2}\left(\rho_{\mathrm{j}-1,0 \lambda 3} / 2\right)\right]\right\} \\
& /\left\{2\left[\cos ^{2}\left(\rho_{\mathrm{j}-1,0 \lambda 1} / 2\right)+\cos ^{2}\left(\rho_{\mathrm{j}-1,0 \lambda 2} / 2\right)+\cos ^{2}\left(\rho_{\mathrm{j}-1,0 \lambda 3} / 2\right)\right]\right\} .
\end{aligned}
$$

Here, the term []$^{0.5}{ }_{\lambda \mathrm{i}}$ in the denominator represents the term at $\lambda_{\mathrm{i}}$.

The unwrapping of $\rho_{\text {jtot } \lambda \mathrm{i}}$ can be executed based on the continuity of $\rho_{\mathrm{jtot}} \lambda_{\mathrm{i}}$. In evaluating the continuity of $\rho_{\mathrm{jtot} \lambda \mathrm{i}}$, the unwrapping process is performed by eliminating phase jumps for $\rho_{\mathrm{j} \lambda \mathrm{i}}$, and $\rho$ jtot $\lambda \mathrm{i}$ is determined by adding a constant value for connected $\rho_{\lambda \mathrm{i}}$. When $\rho_{\text {jtot } \lambda \mathrm{i}}$ at a point on the model is known, the constant value at the point is found by comparing $\rho_{\mathrm{jtot} \lambda \mathrm{i}}$ with the connected $\rho_{\mathrm{j} \lambda \mathrm{i}}$. Furthermore, the $\rho_{\mathrm{jtot} \lambda \mathrm{i}}$ at the point can be also determined from values of $\rho_{\mathrm{j} \lambda 1}, \rho_{\mathrm{j} \lambda 2}$, and $\rho_{\mathrm{j} \lambda 3}$ [28].

\section{Measurement of Stokes parameter by the judicious choice of azimuthal settings of a quarter-wave plate and a linear polarizer}

The Stokes vector $S\left(y_{\mathrm{j}}, y_{0} ; \theta\right) \lambda_{\mathrm{i}}$ at $\lambda_{\mathrm{i}}$ can be calculated from the scattered light intensity $I_{\mathrm{j}}\left(\theta, \beta_{2}, \alpha_{2}\right) \lambda_{\mathrm{i}}$, measured under the following azimuth settings (azimuth values in degrees) of $Q_{2}$ and $P_{2}$ as [28-30]

$$
\boldsymbol{S}\left(y_{\mathrm{j}}, y_{0} ; \theta\right)_{\lambda \mathrm{i}}=\left[\begin{array}{c}
S_{0}\left(y_{\mathrm{j}}, y_{0} ; \theta\right)_{\lambda \mathrm{i}} \\
S_{1}\left(y_{\mathrm{j}}, y_{0} ; \theta\right)_{\lambda \mathrm{i}} \\
S_{2}\left(y_{\mathrm{j}}, y_{0} ; \theta\right)_{\lambda \mathrm{i}} \\
S_{3}\left(y_{\mathrm{j}}, y_{0} ; \theta\right)_{\lambda \mathrm{i}}
\end{array}\right]=\left[\begin{array}{c}
{\left[S_{1}\left(y_{\mathrm{j}}, y_{0} ; \theta\right)^{2}+S_{2}\left(y_{\mathrm{j}}, y_{0} ; \theta\right)^{2}+S_{3}\left(y_{\mathrm{j}}, y_{0} ; \theta\right)^{2}\right]^{0.5}} \\
I_{\mathrm{j}}(\theta, 0,0)_{\lambda \mathrm{i}}-I_{\mathrm{j}}(\theta, 90,90) \lambda_{\mathrm{i}} \\
I_{\mathrm{j}}(\theta, 45,45)_{\lambda \mathrm{i}}-I_{\mathrm{j}}(\theta, 135,135)_{\lambda_{\mathrm{i}}} \\
{\left[I_{\mathrm{j}}(\theta,-45,0)_{\lambda \mathrm{i}}-I_{\mathrm{j}}(\theta, 45,0)_{\lambda \mathrm{i}}\right] / \cos \Delta \rho_{\lambda_{\mathrm{i}}}}
\end{array}\right],
$$

where $I_{\mathrm{j}}\left(\theta, \beta_{2}, \alpha_{2}\right) \lambda_{\mathrm{i}}$ is the light intensity for $\lambda_{\mathrm{i}}$ measured from the scattered light at point $y_{\mathrm{j}}$, and $\theta$ is the azimuthal angle of the polarization axis of the scattered light measured with respect to the $\mathrm{z}$-axis. In addition, $\alpha_{2}$ is the azimuthal angle of the fast axis of $P_{2}$ and $\beta_{2}$ is the azimuthal angle of the transmission axis of $Q_{2}$, while $\Delta \rho_{\lambda_{\mathrm{i}}}$ is the phase difference error by the mismach of $Q_{2}$ with respect to $\lambda_{\mathrm{i}}$. According to Eq. (22), the Stokes parameters at $\lambda_{\mathrm{i}}$ are unaffected by $\Delta \rho_{\lambda_{\mathrm{i}}}$ of $Q_{2}$ due to $\lambda_{\mathrm{i}}$.

\section{Experiments}

\subsection{Experimental system and experimental results}

In the experimental system, a beam having three wavelengths ( $\left.\lambda_{1}=632.8 \mathrm{~nm}, \lambda_{2}=514.5 \mathrm{~nm}, \lambda_{3}=457.9 \mathrm{~nm}\right)$ passes through $P_{1}$, and an electro-optical modulation device EO orthogonally modulates the polarized light at $500 \mathrm{~Hz}$. The light beam can therefore be approximately regarded as an unpolarized beam of light over the long exposure time of the CCD camera. The light beam is then converted into a light sheet, and is split into two light sheets having angles of incidents of $0^{\circ}$ and $45^{\circ}$. The light scattered from the model passes through $Q_{2}$ of wavelength 515 $\mathrm{nm}$ and $P_{2}$, and is imaged by the CCD camera. The phase

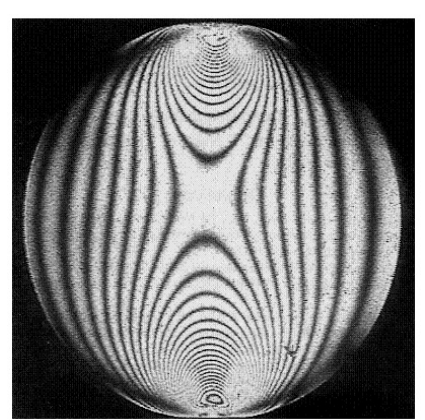

Fig.2. Isochromatics and isoclinics of a frozen stress sphere under diametral compression as measured using a crossed-plane polariscope. 
difference errors of $Q_{2}$ for $\lambda_{1}, \lambda_{2}$ and $\lambda_{3}$ were $\Delta \rho_{\lambda 1}=-17^{\circ}$, $\Delta \rho_{\lambda 2}=0^{\circ}$ and $\Delta \rho_{\lambda 3}=11^{\circ}$, respectively. However, it is important to note that $\psi_{\mathrm{j}}$ and $\rho_{\mathrm{j} \lambda \mathrm{i}}$ determined from Eq.(24) are unaffected by $\Delta \rho_{\lambda \mathrm{i}}[30]$.

A spherical frozen-stress model of diameter $2 R=50.1$ $\mathrm{mm}$ as shown in Fig. 2 was immersed in a fluid that had a refractive index which closely matched that of the sphere. The model was optically sliced into four planes between the top point of the model and its meridian plane (see Fig. 3 ). Thus, the secondary principal axes of the optical slices have been rotated [25]. The original 12 images were

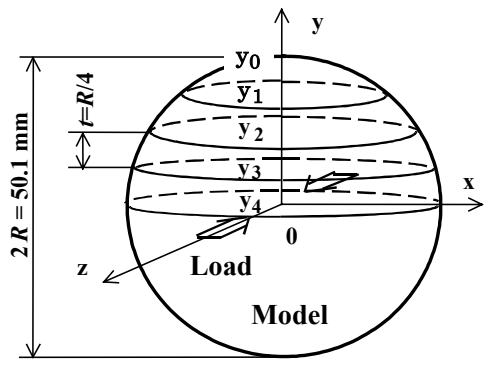

Fig.3. 6.3-mm-thick slices obtained by optical slicing of a frozen stress sphere.

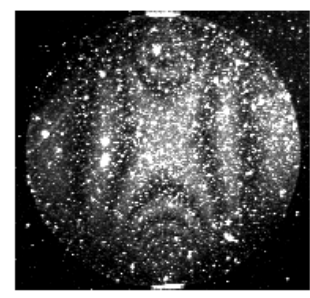

(a)

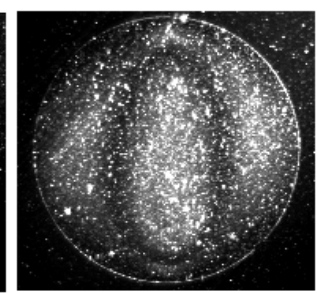

(b)

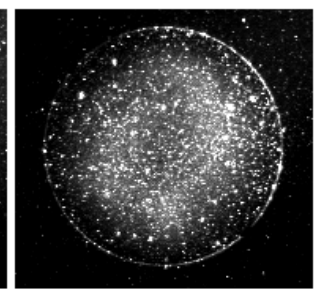

(c)

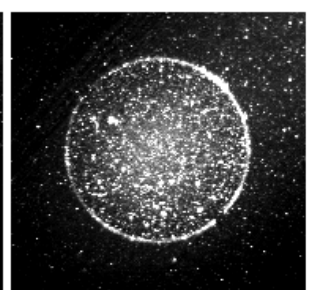

(d)

Fig.4. Original images of $I_{j}(45,135,135){ }_{\lambda 1}$ measured from each section. (a) $I_{4}(45,135,135){ }_{\lambda 1}$, (b) $I_{3}(45,135,135)$ $\lambda_{1}$, (c) $I_{2}(45,135,135) \lambda_{\lambda 1}$, and (d) $I_{1}(45,135,135){ }_{\lambda 1}$.

obtained for each wavelength $\lambda_{\mathrm{i}}$ and for each cross section and were recorded using two incident unpolarized light sheets of $0^{\circ}$ and $45^{\circ}$. Figure 4 shows the original images $I_{\mathrm{j}}$ $(45,135,135)_{\lambda 1}$ measured from each section. The images $I_{\mathrm{j}}$ $(45,135,135)_{\lambda 1}$ contain many speckles due to the dark current of the CCD camera head caused by long time exposures.

\subsection{Analytical Results}

The procedure for determining $\psi_{4}$ and $\rho_{4}$ of the fourth

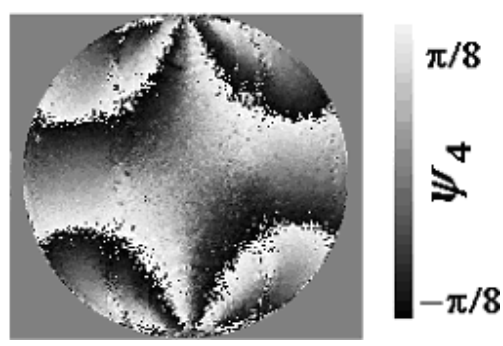

Fig.5. Image of $\psi_{4}$ obtained by using three wavelengths. optical slice, $M_{4}$, is first described. The normalized polarization vectors $s\left(y_{4}, y_{0} ; 0\right)_{\lambda_{\mathrm{i}}}$ and $s\left(y_{4}, y_{0} ; 45\right) \lambda_{\mathrm{i}}$ from the $y_{4}$ section were calculated using Eq. (24) from the original images $I_{4}\left(0, \beta_{2}, \alpha_{2}\right)_{\lambda_{\mathrm{i}}}$ and $I_{4}\left(45, \beta_{2}, \alpha_{2}\right)_{\lambda \mathrm{i}}$, respectively. Similarly, the vectors $s\left(y_{3}, y_{0} ; 0\right)_{\lambda \mathrm{i}}$ and $s\left(y_{3}, y_{0} ; 45\right)_{\lambda \mathrm{i}}$ from the $y_{3}$ section were calculated using the original images $I_{3}\left(0, \beta_{2}, \alpha_{2}\right) \lambda_{\mathrm{i}}$ and $I_{3}\left(45, \beta_{2}, \alpha_{2}\right) \lambda_{\mathrm{i}}$.

The image of $\psi_{4}$ was calculated from Eq. (21) using the three wavelengths, since $\psi_{4}$ obtained from Eq. (19) was not measured at positions for which $\rho_{4 \text { tot } \lambda \mathrm{i}}=2 \pi N_{\lambda \mathrm{i}}$. Figure 5 shows an image of the calculated $\psi_{4}$, and it has abrupt jumps of $\pi / 4$. The obtained $\psi_{4}$ compensates well for the influence of the positions of $\rho_{4 \text { tot } \lambda_{\mathrm{i}}}=2 \pi N_{\lambda_{\mathrm{i}}}$, although it contains many speckles in the scattered light. The values of $\psi_{4}$ were then unwrapped to extend the range to $[-\pi / 2, \pi / 2]$ and the image of the unwrapped $\psi_{4}$ is shown in Fig. 6(a). The unwrapped value of $\psi_{4}$ was easily determined by just adding a constant $\pi / 4$ or $-\pi / 4$ to eliminate the abrupt jumps. In a similar manner, the images of unwrapped $\psi_{\mathrm{j}}$ for the other slices are shown in Figs. 6(b) - (d).

To obtain $\rho_{4}$ at $\lambda_{1}, \rho_{4 \lambda 1}$, the values of $\sin 2 \omega_{3,0}$ and $\cos 2 \omega_{3,0}$ were calculated using Eqs. (22) and (23) for the three wavelengths, respectively. The images of the calculated $\sin 2 \omega_{3,0}$ and $\cos 2 \omega_{3,0}$ are shown in Figs. 7(a) and (b). Figures 7(a) and (b) show that the principal stress axes are rotated 


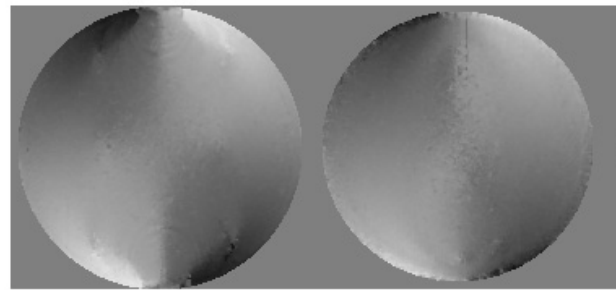

(a) (b)

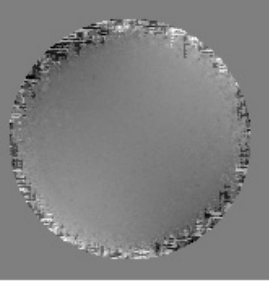

(c)

(d)

Fig. 6. Images (a) to (d) show unwrapped $\psi_{\mathrm{j}}$ of the optical slices $4,3,2$, and 1, respectively.

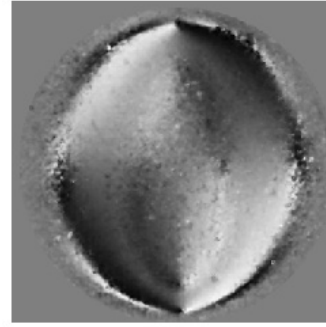

(a)

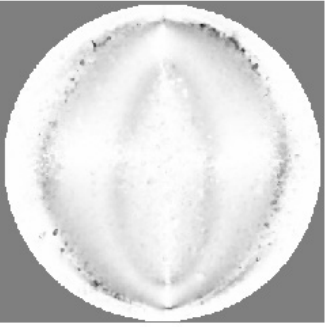

(b)

Fig. 7. Images of obtained (a) $\sin 2 \omega_{3,0}$, (b) $\cos 2 \omega_{3,0}$.

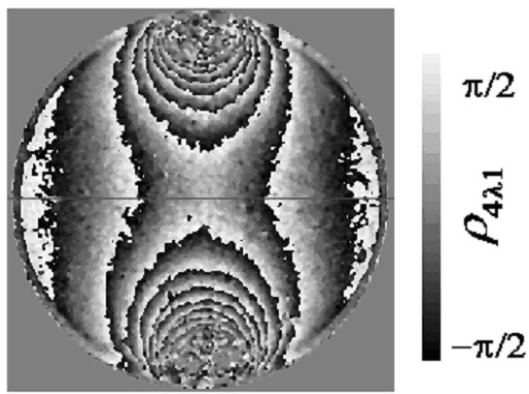

Fig. 8. Images of $\rho_{4 \lambda 1}$ for optical slice 4 .
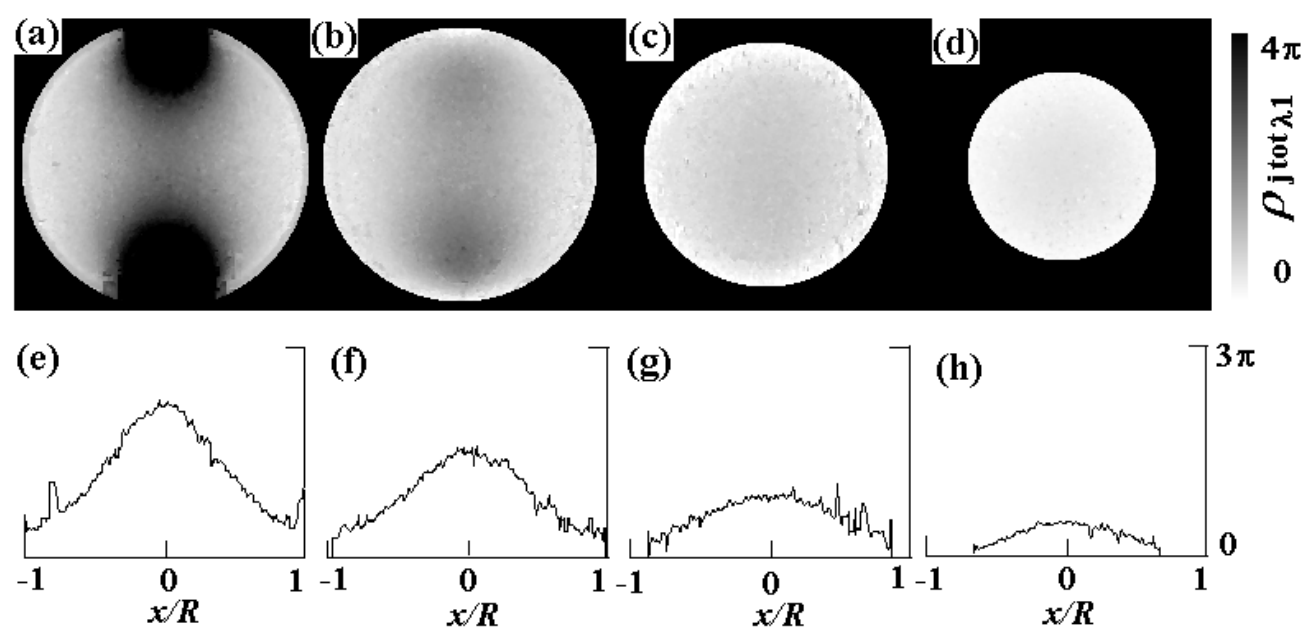

(h)

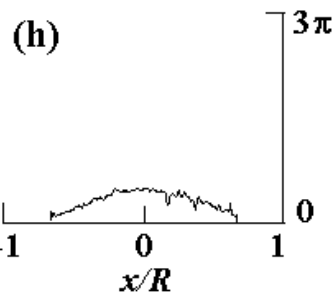

Fig. 9. Images (a) to (d) show unwrapped $\rho_{\text {. j } \lambda 1}$ of the optical slices $4,3,2$, and 1 , respectively. (e) - (h) distributions of unwrapped $\rho_{\mathrm{j} \lambda 1}$ on equatorial plane.

everywhere except in the equatorial and meridian planes. This is a characteristic of the spherical frozen stress model [22]. Then, $\rho_{4 \lambda 1}$ was calculated using Eq. (20) by using $s_{1}\left(y_{3}, y_{4} ; 0\right) \lambda_{11}, s_{3}\left(y_{3}, y_{4} ; 0\right) \lambda_{11}$, $s_{2}\left(y_{3}, y_{4} ; 45\right)_{\lambda 1}, s_{3}\left(y_{3}, y_{4} ; 45\right) \lambda_{\lambda 1}$, and the known values of unwrapped $\psi_{4}$. Figure 8 show the image of $\rho_{4 \lambda 1}$ and the distribution of $\rho_{4 \lambda 1}$ exhibits abrupt jumps of $\pi$. Since the retardation of the model varies continuously, the values of $\rho_{4 \lambda 1}$ were made continuous by eliminating the phase jumps, either $\pi$ or $\pi$, from the center of the model. This connected $\rho_{4 \lambda 1}$ differs from $\rho_{4 \text { tot } \lambda 1}$ by a constant value of $2 \pi$. Thus, the unwrapped $\rho_{4 \operatorname{tot} \lambda 1}$ was obtained by adding $2 \pi$ to the connected $\rho_{4 \lambda 1}$ and is shown in Fig. 9(a) for the range $[0,4 \pi]$. In a similar manner, the images of the unwrapped $\rho_{\operatorname{jtot} \lambda 1}$ for the other 
slices' $\rho_{\mathrm{j} \lambda 1}$ are shown in Figs. 9(b)-(d). The distributions of the unwrapped $\rho_{\mathrm{jtot} \lambda 1}$ in the equatorial plane are shown in Figs. 9(e-h). The images of the measured $\rho_{\text {jtot } \lambda 1}$ are clear, although speckles and the nonuniformity of the scattered light are visible.

These optical measurement results are sufficiently accurate compared with the mechanical slice models that was physically cut from the frozen stress sphere [19].

\section{Conclusions}

The principle of the digital scattered-light photoelasticity with unpolarized light is intelligibly presented, which can be used to nondestructively measure the secondary principal stress direction $\psi_{\mathrm{j}}$ and the total relative phase retardation $\rho_{\text {jtot }}$ in a three-dimensional stressed model with rotation of the principal stress axes. In the present method, the unwrapping of $\psi_{\mathrm{j}}$ and $\rho_{\mathrm{j} \text { tot } \lambda \mathrm{i}}$ for the perfectly automatic stress analysis was obtained easily by using the phase unwrapping method that employs the arctangent function. The measured values of $\psi_{\mathrm{j}}$ and $\rho_{\mathrm{j} \lambda \mathrm{i}}$ are not affected by the phase difference error of the quarter-wave plate. The usefulness of the method then was shown for measurement of the $\psi_{\mathrm{j}}$ and $\rho_{\mathrm{j} \text { tot } \lambda \mathrm{i}}$ in a frozen stress sphere model.

\section{References}

1. H. Hurwitz, and R.C. Jones, J.Opt.Soc.Am.,31,493-499(1941).

2. H.K. Aben, Exp. Mech., 6(1),13-22(1966).

3. L.S. Srinath, and A.V.S.S.S.R. Sarma, Exp. Mech., 14(3),118-122 (1974).

4. P.S. Theocaris, and E.E. Gdoutos, Matrix Theory of Photoelasticity ( Springer-Verlag, 1979 ).

5. R.A. Tomlinson, and E.A. Patterson, Exp. Mech., 42, 43-49(2002).

6. H. Aben, and A. Errapart, in Advance in Experimental Mechanics, (C. Pappalettere, ed., McGrawHill 2004), paper No. 60, ICEM12, 29 August-2 September, 2004 Politecnico di Bari, Italy.

7. R. Weller, J. Appl. Phys.,10, 266(1939).

8. H.J. Menges, Z. Angew. Math. Mech., 20, 210-217(1940).

9. H.T.Jessop, Bri. J. Appl. Phys.,2, 249-260(1951).

10. M.M. Frocht, and L.S. Srinath, Proc. 3rd U.S. Nat. Congr. Appl. Mech.,329-337(1958).

11. Y. F. Cheng, Exp. Mech., 9, 407-412(1969).

12. A. Robert, and E. Guillemet, Brit. J. Appl. Phys., 15, 567-578(1964).

13. A. Robert, Exp. Mech., 7(5), 224-232(1967).

14. J.F. Gross-Petersen, Exp. Mech., 317-322(1974).

15. T. Kihara, H. Kubo, and R. Nagata, Appl.Opt.,18(3),321-327(1979).

16. T. Kihara, M. Unno, C. Kitada, H. Kubo, and R. Nagata, Appl.Opt.,26(4),643-649(1987).

17. T. Kihara, Exp. Mech., 37(1),39-44 ( 1997).

18. T. Kihara, Proceedings of the Japan Society for Photoelasticity,18(1),15-19 ( 1998).

19. T. Kihara, Journal of the Japanese Society for Experimental Mechanics, 4(1), 22-28 ( 2004).

20. T. Kihara, Exp. Mech., 44(5),455-460 ( 2004).

21. T. Kihara, Appl.Opt., 45(35), 8848-8854(2006).

22. T. Kihara, Appl.Opt., 46(25), 6469-6475(2007).

23. R. Desailly, Opt. Commun., 19(1), 61-64(1976).

24. J.C. Dupré and A. Lagarde, Exp. Mech., 37(4),393-397(1997).

25. M.M. Frocht and R.J. Guernesy, NACA Tech. Note 2822(1952).

26. W.H. McMaster, Rev. Mod. Phys., 33,8-28(1961).

27. K. Ramesh, Digital Photoelasticity, Springer-Verlag. (2000).

28. T. Kihara, Strain., 39(2), 65-71(2003).

29. T. Kihara, Opt. Commun., 110, 529-532(1994).

30. T. Kihara, Advance in Experimental Mechanics IV, (J.M. Dulieu-Barton and S. Quinn, Trans Tech Publications LTD, 235-240, 2005). 